\title{
Pleiotropic function of the SQUAMOSA PROMOTER-BINDING PROTEIN-LIKE gene TaSPL14 in wheat plant architecture
}

\author{
Jie Cao ${ }^{1}$ Kaiye Liu ${ }^{1} \cdot$ Wanjun Song $^{1} \cdot$ Jianing Zhang ${ }^{1} \cdot$ Yingyin Yao ${ }^{1} \cdot$ Mingming Xin ${ }^{1} \cdot$ Zhaorong Hu$^{1} \cdot \mathrm{Huiru}$ Peng ${ }^{1}$. \\ Zhongfu $\mathrm{Ni}^{1}$ - Qixin Sun ${ }^{1}$ · Jinkun $\mathrm{Du}^{1}$ (1)
}

Received: 29 July 2020 / Accepted: 6 December 2020 / Published online: 22 January 2021

(c) The Author(s) 2021

\begin{abstract}
Main conclusion The function of SQUAMOSA PROMOTER-BINDING PROTEIN-BOX gene TaSPL14 in wheat is similar to that of $O S S P L 14$ in rice in regulating plant height, panicle length, spikelet number, and thousand-grain weight of wheat, but differs during tiller development. TaSPL14 may regulate spike development via ethylene-response gene EIN3-LIKE 1 (TAEIL1), ETHYLENE-RESPONSIVE TRANSCRIPTION FACTOR 2.11 (TaRAP2.11), and ETHYLENERESPONSIVE TRANSCRIPTION FACTOR 1 (TaERF1), but not DENSE AND ERECT PANICLE 1 (TaDEP1) in wheat.
\end{abstract}

\begin{abstract}
The SQUAMOSA PROMOTER-BINDING PROTEIN-LIKE gene OSSPL14 from rice is considered to be a major determinant of ideal plant architecture consisting of few unproductive tillers, more grains per spike, and high resistance of stems to lodging. However, the function of its orthologous gene, TaSPL14, in wheat is unknown. Here, we reported the functional similarities and differences between TaSPL14 and OSSPL14. Similar to OsSPL14 knock-outs in rice, wheat TaSPL14 knock-out plants exhibited decreased plant height, panicle length, spikelet number, and thousand-grain weight. In contrast to OsSPL14, however, TaSPL14 did not affect tiller number. Transcriptome analysis revealed that the expression of genes related to ethylene response was significantly decreased in young spikes of TaSPL14 knock-out lines as compared with wild type. TaSPL14 directly binds to the promoters of the ethylene-response genes TaEIL1, TaRAP2.11, and TaERF1, and promotes their expression, suggesting that TaSPL14 might regulate wheat spike development via the ethylene-response pathway. The elucidation of TaSPL14 will contribute to understanding of the molecular mechanisms that underlie wheat plant architecture.
\end{abstract}

Keywords Ethylene response genes · Panicle development · Plant height · TaSPL14 · Thousand-grain weight · Tiller number $\cdot$ Wheat

\section{Abbreviations}

IPA1 Ideal Plant Architecture 1

SBP SQUAMOSA-BINDING PROTEIN

SPL SQUAMOSA PROMOTER-BINDING PROTEIN-LIKE

Communicated by Dorothea Bartels.

Jie Cao and Kaiye Liu have contributed equally to this work.

Supplementary Information The online version contains supplementary material available at https://doi.org/10.1007/s0042 5-020-03531-X.

Jinkun Du

jkdu@cau.edu.cn

Extended author information available on the last page of the article

\section{Introduction}

The rice SQUAMOSA PROMOTER-BINDING PROTEINLIKE (SPL) gene OsSPL14 (also known as Ideal Plant Architecture 1, IPAl) encodes a plant-specific transcription factor and is a new "Green Revolution" gene that plays a critical role in regulating rice ideal plant architecture (Wang and Wang 2017; Wang and Zhou 2018; Liu et al. 2019). Therefore, the optimally modulated expression of OsSPL14 in rice could confer ideal plant architecture, including reduced tiller number, increased panicle primary branching, elevated lodging resistance of stems, and increased thousand-grain weight (Kerr and Beveridge 2017; Song et al. 2017; Zhang et al. 2017). Three natural alleles of OSSPL14 have been identified from different high-yield rice lines. The ipa $1-1 D$ allele contains a nucleotide substitution (C to A) that affects miR156 targeting, leads to increased expression of OsSPL14 in the 
shoot apex, and generates ideal plant architecture (Jiao et al. 2010; Wang et al. 2017). Reduced DNA methylation in the promoter region of the WEALTHY FARMER'S PANICLE $(W F P)$ epigenetic allele shows an optimally increased expression of OSSPL14 in young spikes, which strongly positively affects primary branch and grain number in rice (Miura et al. 2010). The ipal-2D epigenetic allele exhibits an elevated expression of OSSPL14 due to the presence of tandem repeat sequences in the promoter region, which leads to an open chromatin structure that controls tiller number, stem diameter, and panicle primary branch number in a dose-dependent manner (Zhang et al. 2017). In addition, base deletion in the exon of OSSPL14 by gene editing causes a frameshift mutation, and the inactivation of OsSPL14 leads to a dwarf phenotype, with an extremely increased number of tillers, decreased plant height, panicle length, and spikelet number (Li et al. 2016). Collectively, these findings demonstrate that OsSPL14 plays an important role in regulating ideal plant architecture in rice.

It was previously reported that OsSPL14 regulates ideal plant architecture by directly binding the GTAC core motif in the promoter sequences of target genes and controlling their expression (Lu et al. 2013). One such target gene, DENSE AND ERECT PANICLE1 (DEP1), controls rice panicle size (Huang et al. 2009; Zhao et al. 2016). A higher expression of OSSPL14 led to a significantly and slightly increased level of $D E P 1$ expression in shoot apices and young panicles, respectively (Lu et al. 2013). The panicle length of RIL-IPAI/depl lines was significantly shorter than that in RIL-IPAI/DEPI lines, suggesting that OsSPL14 positively regulates $D E P 1$ in determining panicle length in rice (Huang et al. 2009; Lu et al. 2013). OsSPL14 regulates tiller number by directly activating rice TEOSINTE BRANCHED I (OSTB1), which is a negative regulator of tillering in rice (Takeda et al. 2003; Lu et al. 2013). The expression of OSTB1 was significantly higher in axillary buds of NIL-ipal plants, which highly express OsSPL14, and the RIL-ipal/tb1 genotype could suppress the tiller phenotype of NIL-ipal plants, indicating that the direct activation of $O s T B 1$ by OsSPL14 contributes to the low number of tillers in NIL-ipal plants (Takeda et al. 2003; Minakuchi et al. 2010; Lu et al. 2013).

In wheat, only few genes have been reported to affect traits related to plant architecture, including tiller inhibition 1 (tin1), tiller inhibition 2 (tin2), tiller inhibition 3 (tin3), and fertile tiller inhibition (ftin), which inhibit tiller formation (Peng et al. 1999; Spielmeyer and Richards 2004; Kuraparthy et al. 2007; Zhang et al. 2013), and the $Q$ gene, which is associated with spike compactness (Feng et al. 2017). However, the effects of the OSSPL14 ortholog TaSPL14 on wheat plant architecture and yield remain largely unknown. The aim of this study was to analyze the function of TaSPL14 in influencing wheat ideal plant architecture. We constructed TaSPL14 knock-out (taspl14) lines using clustered regularly interspaced short palindromic repeats/CRISPR-associated protein 9 (CRISPR/Cas9), and found that taspl14 plants had reduced plant height, panicle length, spikelet number, and thousand-grain weight compared with wild type, but a similar number of tillers. Comparison of the transcriptomes of young spikes of taspl14 and wild-type plants indicated that genes involved in ethylene response were downregulated in TaSPL14 knock-out lines, including TaEIL1, TaERF1, and TaRAP2.11. Furthermore, TaSPL14 directly activated the expression of these three genes in transactivation assays. These functions of TaSPL14 differ from those of OsSPL14. TaSPL14 regulates panicle length of wheat through regulating expression levels of genes associated with ethylene response, but not TaDEP1. Therefore, our data provide insights into the functions and mechanisms of TaSPL14 in the regulation of wheat plant architecture.

\section{Materials and methods}

\section{Plant materials and growth conditions}

Triticum aestivum L. (Fielder) and TaSPL14 knock-out lines (taspl14) were grown in the experimental field of the China Agricultural University in Beijing $\left(39^{\circ} 57^{\prime} \mathrm{N}, 116^{\circ} 17^{\prime} \mathrm{E}\right)$ for seed reproduction, and phenotypic and molecular analyses. Young spikes around $20-30 \mathrm{~mm}$ in length were harvested from wild-type and taspll4 plants, and were immediately frozen in liquid nitrogen and stored at $-80^{\circ} \mathrm{C}$ for subsequent experiments. For molecular analyses, Fielder and taspll4 plants were grown in a controlled environment chamber in conditions of $45 \%$ humidity, $26 / 20^{\circ} \mathrm{C}$ day/night temperature and $16 \mathrm{~h}$ light with $3000 \mathrm{~lx}$ illumination (Master GreenPower CG T 400 W E40, Philips).

\section{Phenotypic analysis}

Plant height, panicle length, spikelet number, mean internode length (the ratio between panicle length and spikelet number), and thousand-grain weight were measured as the mean value for three independent $\mathrm{T}_{2}$ TaSPL14 knock-out lines, \#5-3, \#12-4, and \#13-4, during the harvest stage. Each independent line consisted of at least seven plants. Tiller number was measured at the filling stage, and was calculated as the mean value for 10 different plants from each knock-out line.

\section{Identification of TaSPL genes in wheat}

To identify $S P L$ gene family members in wheat, we downloaded the sequence of SQUAMOSA-PROMOTER-BINDING PROTEIN (SBP domain, PF03110) from Pfam (http:// pfam.xfam.org/). All sequences that contained an SBP domain were obtained from the Triticum aestivum IWGSC 
database (http://plants.ensembl.org/biomart/) based on a hidden Markov model (HMM) profile with a cut-off $E$ value of $<1 \times 10^{-5}$ (Finn et al. 2011; Zhang et al. 2014; Clavijo et al. 2017). The presence of a conserved SBP domain in each predicted gene was confirmed using the Conserved Domain Database (CDD) website (http://www.ncbi.nih.gov/ cdd). Analysis of homoeologous genes was performed using the Ensembl Plant website (http://plants.ensembl.org/index .html).

\section{Phylogenetic analysis of SPL genes}

For phylogenetic analysis, the sequences of 19 OsSPL genes were obtained from GRAP (Xie et al. 2006). Phylogenetic analysis was performed using the amino-acid sequence of the encoded SBP domain ( $\mathrm{Li}$ and Lu. 2014). We used the CDD website to confirm the encoded SBP domain aminoacid sequences of the identified SBP-box genes in rice and wheat. Multiple sequences' alignment was performed with the ClustalW algorithm of MEGA5.02 (Li and Lu 2014). A phylogenetic tree was constructed using the neighbor-joining method and bootstrapping with 1000 replicates. The accession numbers of the $O S S P L$ and $T a S P L$ genes are shown in Suppl. Table S1.

\section{Reverse transcription quantitative real-time PCR (RT-qPCR) analysis}

Total RNA was extracted from grains at 4, 15 and 25 days after pollination using the Quick RNA Isolation Kit (Beijing, China, Waryong, 0416-50) according to the manufacturer's instructions, and Trizol (Invitrogen) reagent was used to isolate total RNA from young roots, stems at the jointing stage, and young leaves young spikes $5,10,20$, and $30 \mathrm{~mm}$ in length. The RNA concentration was determined with a NanoDrop 2000 spectrophotometer (Thermo Scientific). Firststrand cDNA was synthesized with an M-MLV Reverse Transcriptase kit (TaKaRa). RT-qPCR was performed using the CFX96 real-time system (Bio-Rad) in $10-\mu \mathrm{L}$ reactions containing $2 \mu \mathrm{L}$ each gene-specific primer, $1 \mu \mathrm{L}$ cDNA template, $2 \mu \mathrm{L} \mathrm{ddH_{2 }} \mathrm{O}$, and $5 \mu \mathrm{L}$ SYBR Premix EXTaq II (TaKaRa). The RT-qPCR conditions were $95{ }^{\circ} \mathrm{C}$ for $5 \mathrm{~min}$, followed by 40 cycles of $95{ }^{\circ} \mathrm{C}$ for $30 \mathrm{~s}, 60^{\circ} \mathrm{C}$ for $15 \mathrm{~s}$, and $72{ }^{\circ} \mathrm{C}$ for $15 \mathrm{~s}$. A melting curve was performed for each sample to confirm the specificity of the reactions. The relative expression level of each gene was calculated using the $2^{-\Delta \mathrm{CT}}$ method and was normalized to the CT value of the wheat TaACTIN gene (TraesCS5B02G124100). RT-qPCR was performed in triplicate for each sample (Zheng et al. 2019). Gene-specific primers are shown in Suppl. Table S2.

\section{Vector construction and wheat transformation}

We used CRISPR/Cas9 to edit the genome sequence of TaSPL14 (Xing et al. 2014), and the E-CRISP Design website (http://www.e-crisp.org/E-CRISP/designcrispr.html) to design specific single-guide RNAs (sgRNA) to the TaSPL14coding sequence. We synthesized two reverse complementary sgRNA sequences with $B s a \mathrm{I}$ cohesive ends, and ligated them to the intermediate vector PCBC-MT1T2 by PCR and subsequently inserted the fragments into the terminal vector pBUE411 as previously described (Xing et al. 2014). Plasmids were transformed into immature embryos of the wheat cultivar Fielder by Agrobacterium-mediated transformation using Agrobacterium strain EHA105. Phosphinothricin was used to screen for positive $\mathrm{T}_{0}$ transgenic plants (Ishida et al. 2015). DNA was isolated from $T_{0}$ transgenic plants and wildtype Fielder using the CTAB method, and target fragments of TaSPL14 were amplified using specific primers flanking the sgRNA. The TaSPL14 amplicons were cloned into vectors and sequenced, and $\mathrm{T}_{0}$ transgenic plants were selected in which TaSPL14-A, TaSPL14-B, and TaSPL14-D were all mutated. We used the same method to identify $\mathrm{T}_{1}$ and $\mathrm{T}_{2}$ homozygous TaSPL14 knock-out plants. The primers for vector construction and TaSPL14 target fragments amplification are shown in Suppl. Table S2.

\section{RNA sequencing}

We collected several young spikes $(20-30 \mathrm{~mm})$ from wildtype Fielder and one TaSPL14 knock-out line (taspl14-\#134) for RNA sequencing (RNA-seq). The extraction method of total RNA from young spikes using Trizol (Invitrogen) according to the manufacturer's instructions, and each RNA sample was divided into two to give two technical replicates. At least $5 \mu \mathrm{g}$ total RNA for each replicate was used to construct a cDNA library using Illumina Poly-A Purification TruSeq library reagents, on a NovaSeq platform. The RNA-seq data were analyzed as previously described (Quinlan and Hall 2010; Joshi and Fass 2011; Kim et al. 2013). The transcripts that were differentially expressed between taspl14-\#13-4 and wild type were characterized with the Bioconductor package edgeR with an absolute value of $\log _{2}$ (fold change) $\geq 1$ and a false discovery rate $<0.05$ as cut-offs (Robinson et al. 2010). The gene ontology (GO) analysis was performed using agriGO v.2.0 with a cut-off of $P<0.05$ (Yan et al. 2017).

\section{Electrophoretic mobility shift assay (EMSA)}

The DNA-binding domain of TaSPL14 was expressed in BL21 Escherichia coli cells (Transgene, Beijing, China) by cloning the full-length open-reading frame of TaSPL14-B (TraesCS5B02G265600) into the BamHI restriction enzyme 
site of pGEX6P-1 to generate a chimeric GST-TaSPL14 protein. Expression of recombinant proteins in Transetta (DE3) E. coli (Transgene) was induced with $0.1 \mathrm{mM}$ isopropyl betaD-thiogalactopyranoside (IPTG) in LB buffer overnight at $16{ }^{\circ} \mathrm{C}$. Cells were harvested, washed, and suspended in $20 \mathrm{~mL}$ PBS buffer (137 mM NaCl, $2.7 \mathrm{mM} \mathrm{KCl,} 10 \mathrm{mM} \mathrm{Na}_{2} \mathrm{HPO}_{4}$, and $2 \mathrm{mM} \mathrm{KH}_{2} \mathrm{PO}_{4}$ ), and then were sonicated for $0.5-1 \mathrm{~h}$ in $30 \mathrm{~mL}$ PBS buffer containing $1 \mathrm{mM}$ phenylmethylsulfonyl fluoride (PMSF), and 1/2 tablet of protease inhibitor cocktail (Roche). When the suspension became clear, cells were centrifuged at $13,000 \mathrm{~g}$ for $30-45 \mathrm{~min}$ at $4{ }^{\circ} \mathrm{C}$, and the supernatant was collected and filtered into a $50-\mathrm{mL}$ tube through a $0.22-\mathrm{mm}$ membrane. The supernatant was incubated with 200- $\mu$ L GST MAG Agarose Beads (Novagen) at $4{ }^{\circ} \mathrm{C}$ and shaken overnight. The GST beads were washed three times with $5 \mathrm{~mL}$ PBS buffer and then mixed with the supernatant. The mixture was incubated with $50 \mathrm{mM}$ Tris- $\mathrm{HCl}(\mathrm{pH}=8.0)$ buffer containing $10 \mathrm{mM}$ reduced glutathione at $4{ }^{\circ} \mathrm{C}$ for at least $7 \mathrm{~h}$ to elute TaSPL14-GST recombinant proteins. The NanoDrop 2000 spectrophotometer (Thermo Scientific) was used to determine the concentration of fusion proteins (Guo et al. 2015, 2018). The probes were labeled at the $5^{\prime}$ end with biotin, and unlabeled probes without biotin modification were also prepared that included competition probes with the same sequence as the labeled probes, and probes with a mutated core motif. All probes were synthesized by Invitrogen, and were made double-stranded by cooling from $100{ }^{\circ} \mathrm{C}$ to room temperature in annealing buffer. The LightShift ${ }^{\circledR}$ Chemiluminescent EMSA Kit (Thermo Scientific) was used for EMSA; the DNA-binding system consisted of $1 \times$ binding buffer $(100 \mathrm{mM}$ Tris, $500 \mathrm{mM} \mathrm{KCl,} 10 \mathrm{mM}$ dithiothreitol at $\mathrm{pH} 7.5), 10 \%$ (v/v) glycerol, $0.5 \mathrm{mM}$ EDTA, $10 \mathrm{mM} \mathrm{ZnCl}_{2}$ and $50 \mathrm{ng} \mu \mathrm{L}^{-1}$ poly dI-dC (Thermo Scientific), $2 \mu \mathrm{L}$ fusion protein, $2 \mu \mathrm{L}$ labeled probes $(100 \mu \mathrm{M})$, and $\mathrm{ddH}_{2} \mathrm{O}$ to a final volume of $20 \mu \mathrm{L}$. Competition analysis was performed by adding fivefold and tenfold molar excesses of the unlabeled probes $(50 \mu \mathrm{M})$ to the binding reaction $5 \mathrm{~min}$ before adding labeled probes. Reactions were incubated for $30 \mathrm{~min}$ at room temperature and were then electrophoresed on $6 \%$ native polyacrylamide gels. The gels were transferred to nylon membranes, and shifted complexes of labeled proteins and free probes were visualized using a chemiluminescence camera (Tanon, Beijing, China) (Guo et al. 2018). The sequences of the probes used for EMSA are listed in Suppl. Table S2.

\section{Transcriptional activity assays in Nicotiana tabacum}

Dual-luciferase reporter assays were performed as described previously (Guan et al. 2014). The $2-\mathrm{kb}$ promoter sequences of TaEIL1 (TraesCS2D02G099400), TaERF1 (TraesCS2D02G414600), and TaRAP2.11 (TraesCS2D02G202100) were amplified from Fielder genomic DNA, and cloned into the BamHI and PstI sites of pGreenII containing the coding sequence of LUC from Renilla reniformis (REN) (Hellens et al. 2005) using the In-Fusion ${ }^{\circledR}$ HD Cloning Kit (Clontech), to create the pGreenII reporters pTaEIL1::LUC, pTaERF $1:: L U C$ and pTaRAP2.11::LUC plasmids. pGreenII vector containing the coding sequence of $R$. reniformis $L U C$ driven by the $35 \mathrm{~S}$ promoter (Hellens et al. 2005) was used as a positive control. The full-length coding sequence of the TaSPL14-B sub-genome was amplified and cloned into the Supper1300 overexpression vector to generate the effector construct 35S::TaSPL14. The following plasmids were introduced into Agrobacterium strain GV3101 (Hellens et al. 2005): Empty vector (Empty Supper1300) + pTaEIL1::LUC, 35S::TaSPL14+pTaEIL1::LUC, empty vector (Empty Supper1300) + pTaERF1::LUC, 35S::TaSPL14 + pTaERF1::LUC, empty vector (Empty Supper1300) + pTaRAP2.11::LUC, 35S::TaSPL14+pTaRAP2.11::LUC. Agrobacterium strains harboring these combinations of reporter plasmids and effector plasmids were co-infiltrated into young leaves of 4-week-old Nicotiana benthamiana plants. After infiltration for $48 \mathrm{~h}$, infected leaves were harvested and the activity of $L U C$ driven by the respective promoters of TaEILI, TaERF1, or TaRAP2.11 was quantified using the Dual-Luciferase Reporter Assay system (Promega) with the Synergy 2 Multi-Detection Microplate Reader (BioTek Instruments). Expression of $L U C$ driven by the $35 \mathrm{~S}$ promoter (35S::REN) was used as an internal control. Normalized data are presented as the ratio of the value of luciferase activity to that of the control 35S::LUC signal from three independent biological samples. The primers for the constructs used for the transcriptional activity assays are shown in Suppl. Table S2.

\section{Results}

\section{Identification of TaSPL14 in wheat}

We annotated 56 sequences from the wheat genome (TGACv1 version) (Appels et al. 2018) that contained the SBP domain. Because common wheat (BBAADD) is a typical hexaploid species derived from three diploid ancestral species, Triticum urartu (AA), Aegilops speltoides (BB), and Aegilops tauschii (DD) (Petersen et al. 2006; Li et al. 2015; Ling et al. 2018; Zhang et al. 2018), the homoeologous genes among these 56 sequences from the three subgenomes were grouped to 19 unique TaSPL genes, 10 of which had been previously isolated and named (Zhang et al. 2014) (Suppl. Table S1). We generated a phylogenetic tree based on the SBP domain of 19 OsSPL proteins from rice and 19 TaSPL proteins from wheat (Cardon et al. 1999; Xie et al. 2006; Yang et al. 2008). The remaining nine genes in the tree were named according to the orthologous genes of rice (Fig. 1a). We identified three homoeologous genes, 
TraesCS5A02G265900, TraesCS5B02G265600, and TraesCS5D02G273900, designated TaSPL14-A, TaSPL14-B, and TaSPL14-D, which showed high amino-acid similarity with OsSPL14. Previous studies suggested that OsSPL14 was the target gene of OsmiR156 (Jiao et al. 2010). To verify whether miR 156 could regulate TaSPL14 in vivo, we performed modified RNA ligase-mediated 5'-rapid amplification of cDNA ends (RLM-5'-RACE), and found that TaSPL14 mRNA was cleaved by miR156 at the tenth nucleotide from the $5^{\prime}$ end (Fig. 1b).

We detected three genes homoeologous to TaSPL14 located on chromosomes 5A, 5B, and 5D, respectively, using genomic DNA of a Chinese Spring (CS) line nullisomic-tetrasomic for chromosome group 5. When the corresponding chromosome was deleted from this CS line, no PCR amplicons could be generated (Fig. 1c). Next, we quantified the expression of TaSPL14-A, TaSPL14-B, and TaSPL14-D by RT-qPCR in different tissues, including young roots (YR), stems at the jointing stage (SJ), young leaves (YL), grains at 4, 15, and 25 days after pollination (GDAP), and young spikes $5 \mathrm{~mm}$ (YS5), $10 \mathrm{~mm}$ (YS10), $20 \mathrm{~mm}$ (YS20), or $30 \mathrm{~mm}$ (YS30) in length (Fig. 1d). The expression levels of TaSPL14-B and TaSPL14-D were higher than that of TaSPL14-A in the tissues examined. TaSPL14-A and TaSPL14-D were mostly expressed in stems and young spikes, whereas TaSPL14-B was abundant in stems, leaves, seeds, and young spikes, suggesting that these three homoeologous genes of TaSPL14 contribute differently to wheat development.

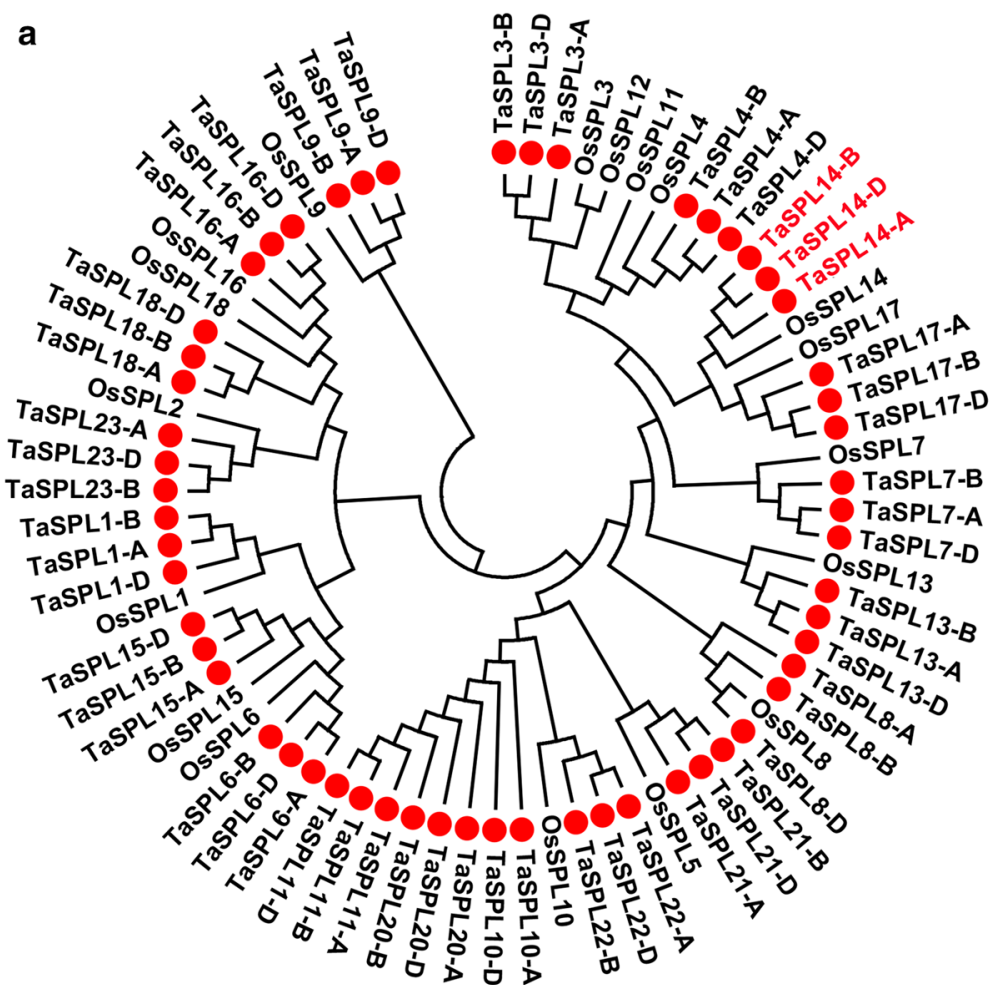

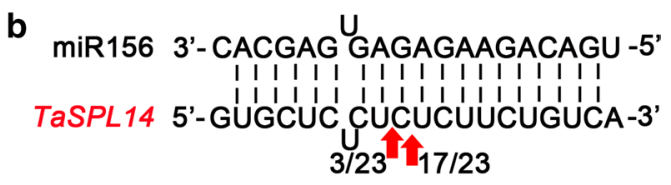
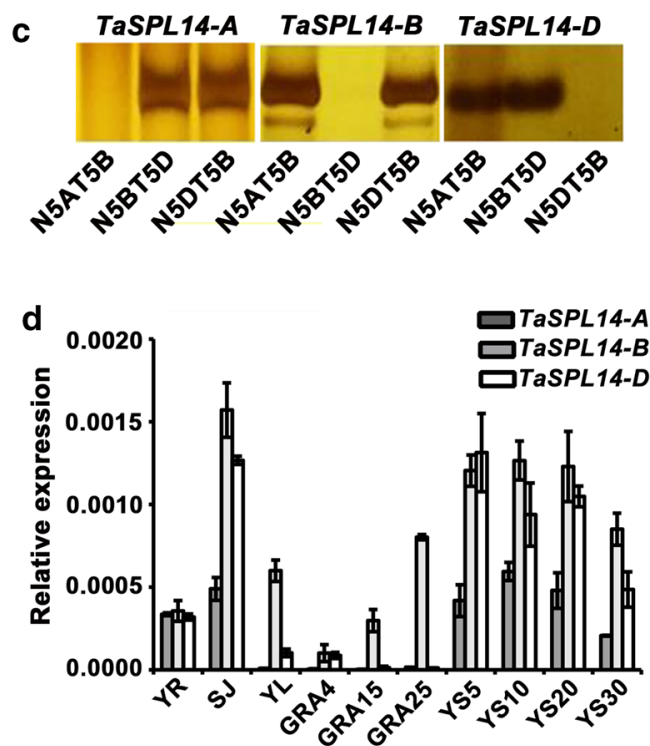

Fig. 1 Identification and expression of TaSPL14 in wheat. a Phylogenetic analysis of SBP-box genes in rice and wheat. The peptide sequences of the SBP domain of OsSPL and TaSPL proteins were aligned using ClustalW, the phylogenetic tree was constructed using MEGA5.02 with the neighbor-joining method, and the bootstrap test was performed with 1000 replicates. b miR156 and TaSPL14 alignment and cleavage frequencies were detected using RLM-5'-RACE. The red arrows indicate the cleavage sites, and the numbers $3 / 23$ and $17 / 23$ show the ratios of the cleaved products from this site out of the total clones confirmed by sequencing. $\mathbf{c}$ The chromosomal locations of three homoeologous TaSPL14 genes. The bands indicate amplification of homoeologous genes from the Chinese Spring (CS) null- tetrasomic lines. The nomenclature represents the different $\mathrm{A}, \mathrm{B}$, or D genomes: for example, N5AT5B represents nullisomic 5A-tetrasomic5B. d The expression of three homoeologous TaSPL14 genes in different tissues: YR, young roots; SJ, stems at the jointing stage; YL, young leaves; GDAP4, GDAP15, and GDAP25 represent grains at 4, 15 , and 25 days after pollination; YS5, YS10, YS20, and YS30 represent young spikes $5,10,20$, or $30 \mathrm{~mm}$ in length. The data for each tissue consist of three biological replications and three technical replicates. The expression levels were normalized to that of wheat $T a A C$ $T I N$. Each bar in the graph corresponds to the mean value of three technical replicates $\pm \mathrm{SD}$ 


\section{TaSPL14 is similar to that of OsSPL14 in regulating plant height, panicle length, spikelet number, and thousand-grain weight of wheat, but differs during tiller development}

To investigate the biological function of TaSPL14 in wheat, we generated TaSPL14 knock-out plants using the CRISPR/ Cas9 system (Xing et al. 2014). To simultaneously knock out the three homoeologous genes TaSPL14-A, TaSPL14-B, and TaSPL14-D, we designed a specific sgRNA that targets the conserved region within the first exon of the TaSPL14$A$, TaSPL14-B, and TaSPL14-D sequences (Fig. 2a). The CRISPR/Cas9 vector $p B U E 411: \because \operatorname{sgRNA}$ was transformed into wild-type Fielder by Agrobacterium-mediated transformation (Ishida et al. 2015). Three independent transgenic $T_{0}$ plants were obtained and DNA sequencing for the TaSPL14 loci revealed that all three homoeologous genes were simultaneously mutated, with frameshift mutations in the proteincoding sequence of TaSPL14 knock-out lines \#5 and \#12, resulting in inactivated TaSPL14 proteins. The TaSPL14 knock-out line \#13 showed a 3-bp deletion in TaSPL14-A that did not cause a frameshift in the protein-coding region, whereas TaSPL14-B and TaSPL14-D each contained a frameshift mutation that produced an inactivated protein (Fig. 2b). Three independent $\mathrm{T}_{2}$ lines, \#5-3, \#12-4, and \#134 , were generated and sown in the field. All three taspll4
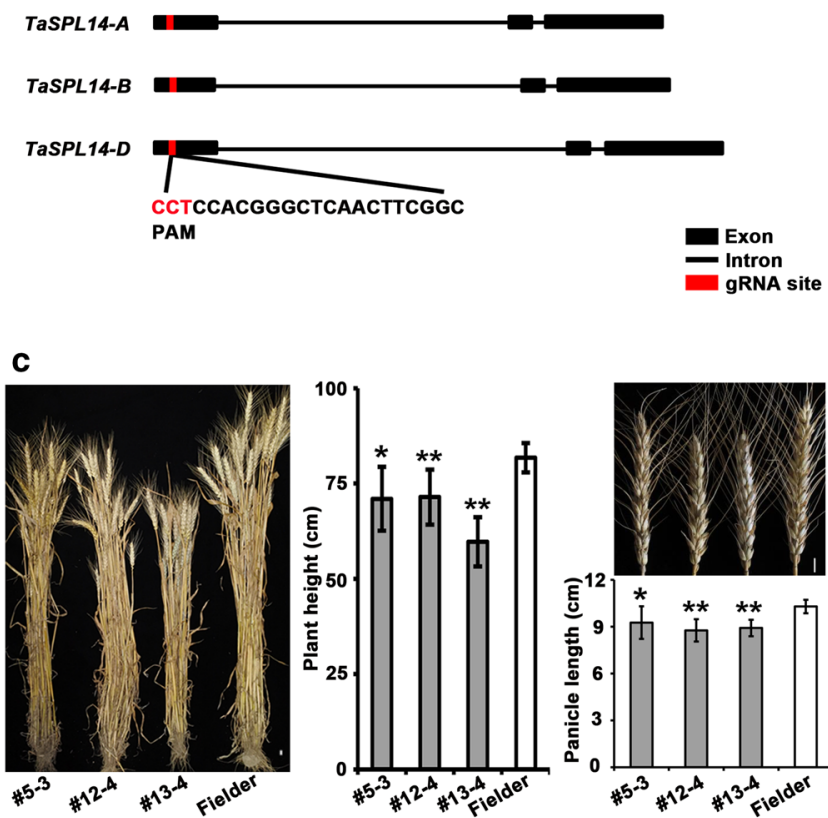

e

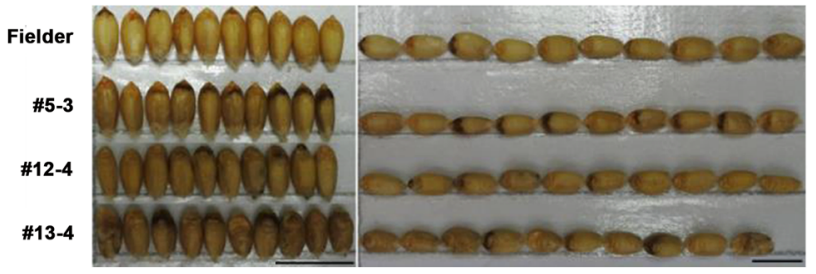

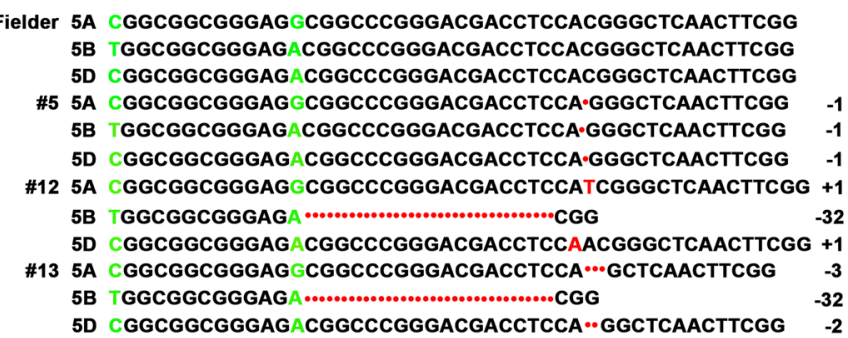

d
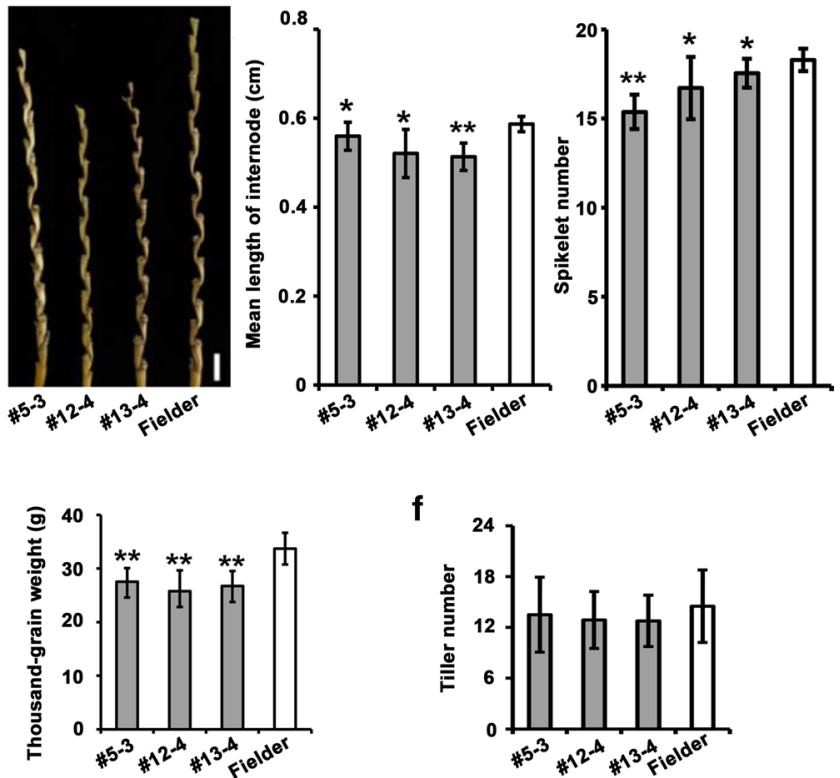

Fig. 2 Phenotypic analysis of TaSPL14 knock-out lines. a Schematic diagrams to illustrate sgRNA design for three TaSPL14 homoeologs. Black boxes and lines represent exons and introns, respectively; red boxes denote the gRNA site. The protospacer-adjacent motif (PAM) sequence is highlighted in red letters, and the sgRNA sequence is in black letters. b The genotypes of three knock-out lines of TaSPL14 were identified by sequencing. The numbers \#5, \#12, and \#13 indicate three independent knock-out lines of TaSPL14; Fielder indicates wild type; " + " and " - " indicate insertions or deletions, respectively, caused by CRISPR/Cas9-induced mutations; numbers indicate the length of the insertion or deletion. $\mathbf{c}$ Plant height and panicle length of TaSPL14 knock-out lines (\#5-3, \#12-4, and \#13-4) and wild type (Fielder). Bar $=1 \mathrm{~cm}$. d Mean internode length and spikelet number of TaSPL14 knock-out lines (\#5-3, \#12-4, and \#13-4) and wild type (Fielder). Bar $=1 \mathrm{~cm}$. e Thousand-grain weight of TaSPL14 knock-out lines (\#5-3, \#12-4, and \#13-4) and wild type (Fielder). $\mathrm{Bar}=1 \mathrm{~cm}$. f Tiller number of TaSPL14 knock-out lines (\#5-3, $\# 12-4$, and \#13-4) and wild type (Fielder). Bar=10 cm. Each graph shows mean values \pm SD for each sample $(n \geq 7)$. Single and double asterisks represent statistically significant differences determined by Student's $t$ test at $P<0.05$ and $P<0.01$, respectively 
lines showed significantly decreased plant height and panicle length compared with wide-type plants (Fig. 2c). Further observations revealed that the mean lengths of the rachis internode and the spikelet number were both significantly lower in taspl14 than in the wild type (Fig. 2d). These results indicate that TaSPL14 influences wheat spike development by affecting both rachis internode elongation and spikelet formation. Thousand-grain weight (TGW) in TaSPL14 knockout lines \#5-3, \#12-4, and \#13-4 was markedly decreased, to $17.3,22.88$, and $23.7 \%$, respectively, of that in wild type, indicating that TaSPL14 influenced grain weight (Fig. 2e). Plant height, panicle length, and the number of flowers in rice osspl14 (ipal) lines were considerably lower than those in wild type ( $\mathrm{Li}$ et al. 2016). Moreover, TGW increased in NIL OsSPL14 ${ }^{\text {ipal }}$, which has a higher expression level of OsSPL14 (Jiao et al. 2010). Thus, our results indicate that TaSPL14 possesses conserved functions with respect to those

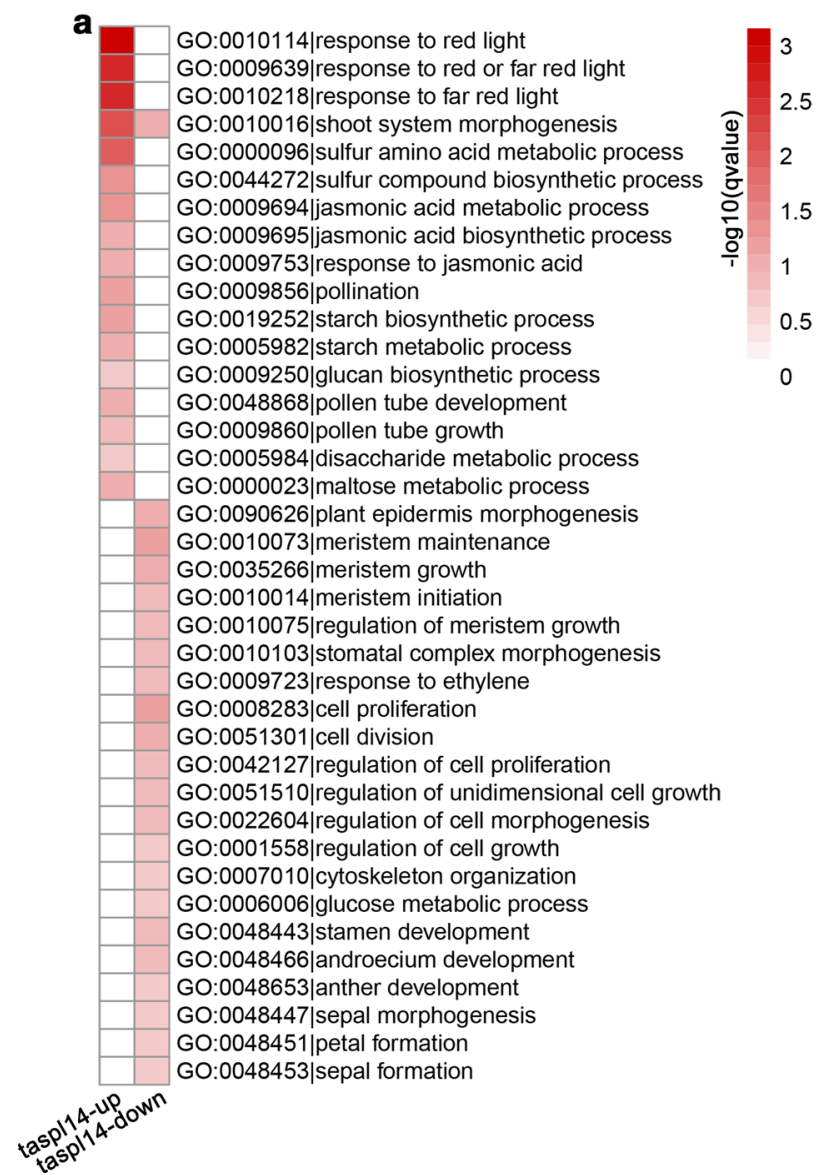

Fig. 3 GO analysis and expression levels of differentially expressed ethylene-response genes in taspl14-\#13-4 and wild-type Fielder. a GO analysis of ethylene-response genes that were differentially expressed in taspl14-\#13-4 and wild type. The color in each cell indicates $\log _{10}(P$ values $)$ of the GO enrichment. b Heat map for the differentially expressed genes involved in ethylene response in the taspl14-\#13-4 line and wild type. C-i Expression levels of differ- of OSSPL14 in that it pleiotropically regulates plant height, panicle length, spikelet number, and TGW weight.

However, in contrast to OSSPL14, which regulates rice tillering (Song et al. 2017), TaSPL14 is not associated with tiller development in wheat. The progeny of tasp14 lines \#5-3, \#12-4, and \#13-4 showed a similar tiller number to wild-type Fielder at the heading stage (Fig. 2f). These data indicate that the function of TaSPL14 in tiller development differs from that of OSSPL14.

\section{TaSPL14 regulated ethylene-response genes, but not TaDEP1 in wheat}

To understand how TaSPL14 regulates spike development, we performed an RNA-seq experiment with young spikes (20-30 mm) from wild-type Fielder and TaSPL14 knock-out line taspl14-\#13-4. TaSPL14-A and TaSPL14-B were not differentially expressed in taspl14-\#13-4 compared with wild

\section{b}
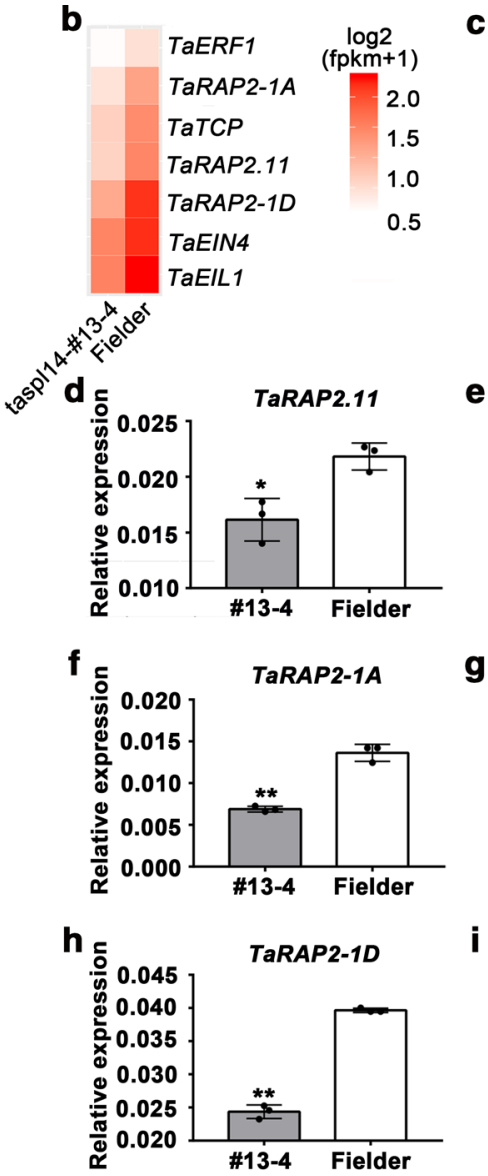
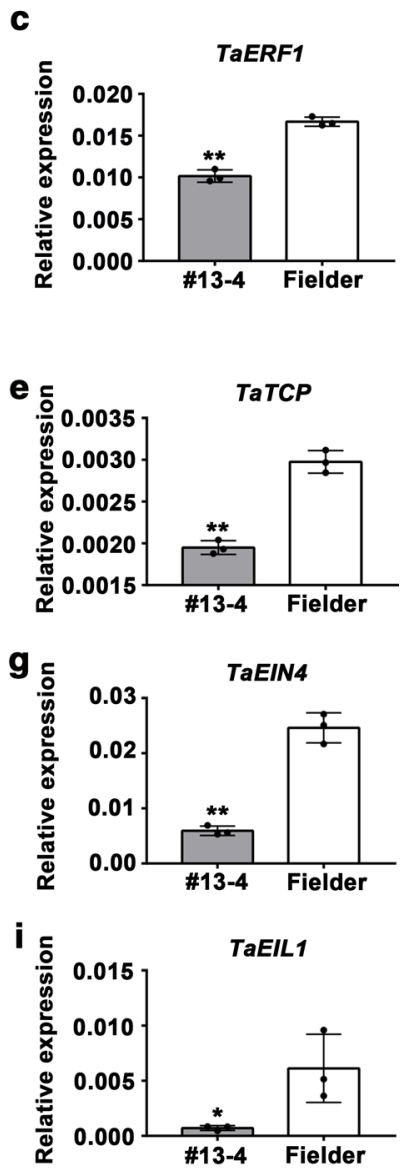

entially expressed genes involved in the ethylene response in young spikes of taspl14-\#13-4 and wild type. The expression level was normalized to that of wheat TaACTIN. Each bar in the graph corresponds to the mean value $\pm \mathrm{SD}$ of three technical replicates, denoted by dots. Single and double asterisks represent statistically significant differences determined by Student's $t$ test at $P<0.05$ and $P<0.01$, respectively 
a

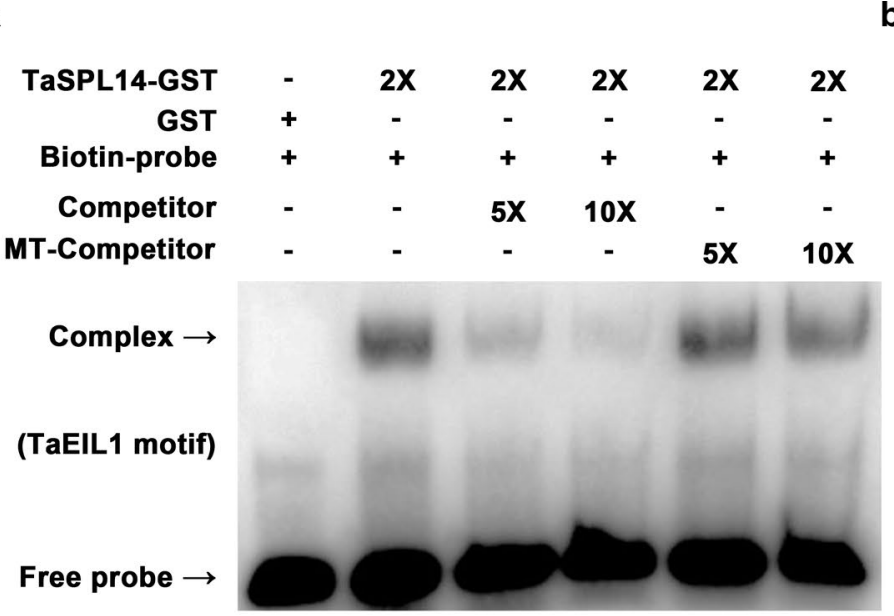

b

Effector

CaMV 35s TaSPL14

Reporter

proTaEIL1 LUC

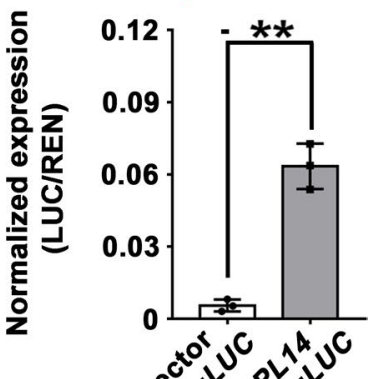

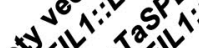

$\times b^{3} \times p$

C

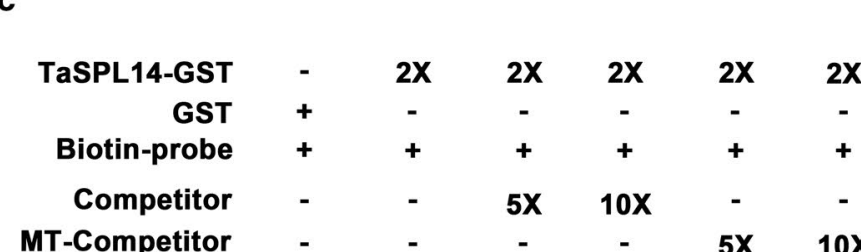

Complex $\rightarrow$

(TaRAP2.11 motif)

Free probe $\rightarrow$

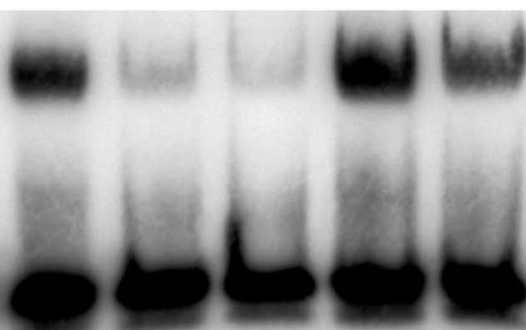

d

Effector
CaMV 35s TaSPL14

Reporter

proTaRAP2.11 LUC

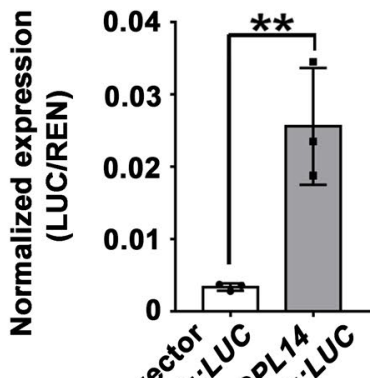

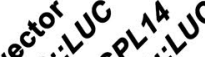

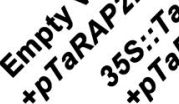

e

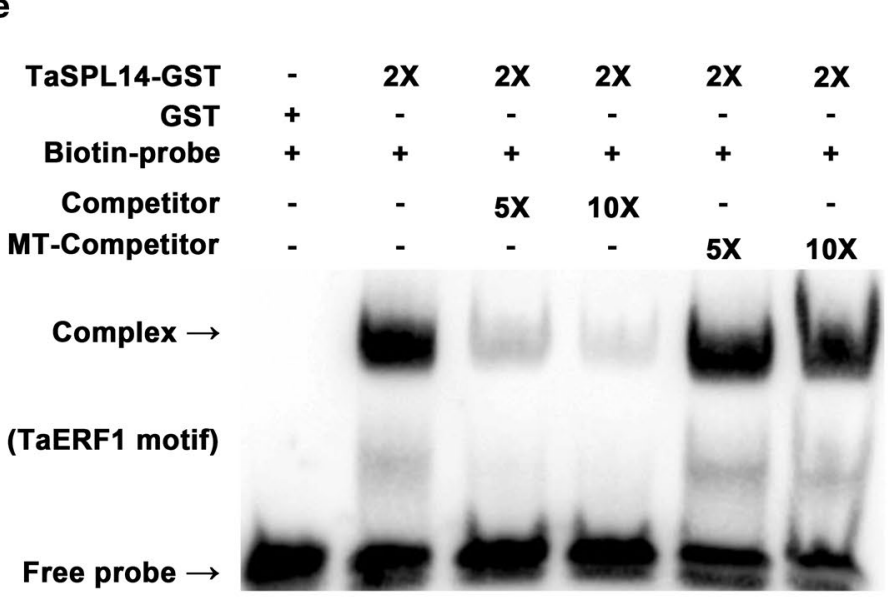

f
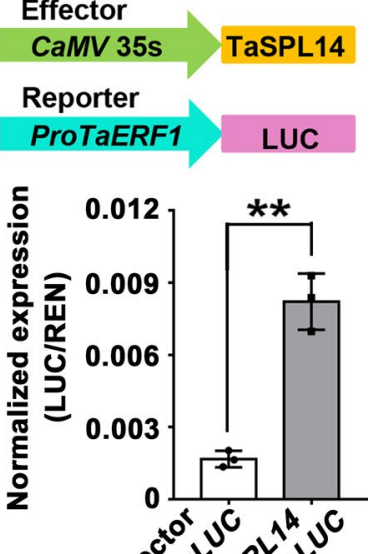

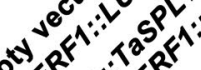


4Fig. 4 TaSPL14 directly binds and activates the promoters of TaEIL1, TaRAP2.11, and TaERF1. a, c, e Results of EMSAs using TaSPL14 recombinant proteins fused to GST and three biotin-labeled probes, competitor probes (biotin-unlabeled), and mutant competitor probes (biotin-unlabeled) derived from the TaEIL1, TaERF1, and TaRAP2.11 promoters, to assess the binding of TaSPL14 to the GTAC core sequence in the promoter regions of TaEIL1, TaRAP2.11, and TaERF1. " " and "-" indicate the presence and absence of corresponding recombinant proteins and probes; " $2 \times$ " indicates the amount of recombinant proteins in the reaction mixtures; " $5 \times$ " and "10x" indicate fivefold and tenfold molar excesses of competitor or mutated competitor (MT) probes relative to the concentration of biotin-labeled probes. b, d, f TaSPL14 directly activated the expression of TaEIL1, TaRAP2.11, and TaERF1. Transactivation assays with Nicotiana benthamiana leaves infiltrated with the effector construct (CaMV35s::TaSPL14) combined with different reporter constructs (proTaEIL1::LUC, proTaRAP2.11::LUC or proTaERF1::LUC). Each bar in the graph corresponds to the mean value \pm SD of three independent replicates, denoted by dots. The double asterisks represent significant differences determined by Student's $t$ test at $P<0.01$

type, and the expression of TaSPL14-D was lower in taspl14\#13-4 than in wild type (Suppl. Fig. S1). In total, 1,103 genes were downregulated and 228 genes were upregulated in taspl14-\#13-4 compared with wild type (Suppl. Table S4). Further analysis of the Gene Ontology groups of differentially expressed genes showed that upregulated genes were mainly enriched in pathways related to responses to red or far-red light and jasmonic acid response, whereas the downregulated genes were mainly enriched in pathways related to meristem maintenance, meristem growth, cell proliferation, and ethylene response (Fig. 3a, Suppl. Table S3).

Among the downregulated genes, we observed that the expression of seven genes associated with ethylene response was statistically significantly lower in taspll4-\#13-4 than in wild type (Fig. 3b): ETHYLENE-RESPONSIVE TRANSCRIPTION FACTOR 1 (TaERF1, TraesCS2D02G414600), ETHYLENERESPONSIVE TRANSCRIPTION FACTOR 2 (TARAP2.11, TraesCS2D02G202100), TEOSINTE BRANCHED1, CYCLOIDEA AND PCF TRANSCRIPTION FACTOR (TaTCP, TraesCS2D02G347200), (ETHYLENE-RESPONSIVE TRANSCRIPTION FACTOR 1 (TaRAP2-1A, TraesCS1A02G231200), ETHYLENE INSENSITIVE 4 (TaEIN4, TraesCS2D02G000500), ETHYLENE-RESPONSIVE TRANSCRIPTION FACTOR 1 (TaRAP2-1D, TraesCS1D02G230900), and EIN3-LIKE 1 (TaEIL1, TraesCS2D02G099400). The downregulation of these genes in taspl14-\#13-4 was validated by RT-qPCR analysis (Fig. 3c-i). Therefore, TaSPL14 regulates the expression of genes related to ethylene response.

OsSPL14 can bind to the GTAC or TGGGCC/T core motif in the promoter regions of its target genes (Lu et al. 2013), which prompted us to analyze whether TaSPL14 regulates genes involved in ethylene responses by binding to their promoters. In the present study, the core GTAC motif was identified within the promoter sequences of TaEIL1, TaRAP2.11, and TaERF1, and we performed electrophoretic mobility shift assays (EMSAs) to determine whether TaSPL14 directly bound these sequences. Specific labeled and unlabeled probes were synthesized from the promoter regions of TaEIL1, TaRAP2.11, and TaERF1. Specific binding was observed between TaSPL14-GST fusion proteins and labeled probes for all three genes, and the EMSA band shift was prevented by the addition of an excessive molar concentration of unlabeled probes, but not mutated unlabeled probes, indicating that TaSPL14 bound the GTAC core motif in the promoters of TaEIL1, TaRAP2.11, and TaERF1 (Fig. 4a-c). To confirm the regulation of TaEIL1, TaRAP2.11, and TaERF1 by TaSPL14 in vivo, we performed transient transcriptional activity assays through Agrobacterium-mediated infiltration of $N$. benthamiana leaves. We constructed three plasmids that contained the $L U C$ reporter gene driven by the promoter sequences of TaEIL1 (pTaEIL1::LUC), TaRAP2.11 (pTaRAP2.11::LUC), or TaERF1 ( $p$ TaERF1::LUC) and an effector plasmid that overexpressed TaSPL14 (35S::TaSPL14) (Fig. 4d-f). The results showed that co-expression of $35 S:: T a S P L 14$ with pTaEIL1::LUC, pTaRAP2.11::LUC, and pTaERF1::LUC led to a significant increase in $L U C$ reporter gene activity compared with that of the empty-vector controls. This indicates that TaSPL14 can directly and dramatically elevate the expression of TaEIL1, TaRAP2.11, and TaERF1 (Fig. 4d-f).

The $O s D E P 1$ gene is directly and positively regulated by OsSPL14, and it is an important regulatory gene that affects panicle architecture (Huang et al. 2009; Lu et al. 2013; Xu et al. 2016). To assess whether the SPL14-DEPI regulatory module is conserved between rice and wheat, we detected the expression of TaDEP1 in taspl14-\#13-4 and wild type. The three homoeologous genes TaDEPI-A (Traes $C$ S5A02G215100), TaDEP1-B (TraesCS5B02G208700), and TaDEP1-D (TraesCS5D02G216900) are present in wheat (Vavilova et al. 2017; Dong et al. 2019), and RNA-seq demonstrated that they were not differentially expressed between young spikes of the TaSPL14 knock-out line taspl14-\#13-4 and the wild type, implying that TaSPL14 regulates spike development via other genes that are related to ethylene response, rather than via TaDEP1 (Suppl. Fig. S2).

\section{Discussion}

The OsSPL14 gene is an essential component of the regulation of ideal rice plant architecture, including tiller development, panicle architecture, and the resistance of stems to lodging, and thereby substantially contributes to grain yield (Lu et al. 2013; Ordonio and Matsuoka 2017). However, the molecular and physiological functions of its ortholog, TaSPL14, in wheat are largely unknown. Therefore, in this study, we investigated the role and mechanisms of TaSPL14 function in regulating wheat plant architecture. 


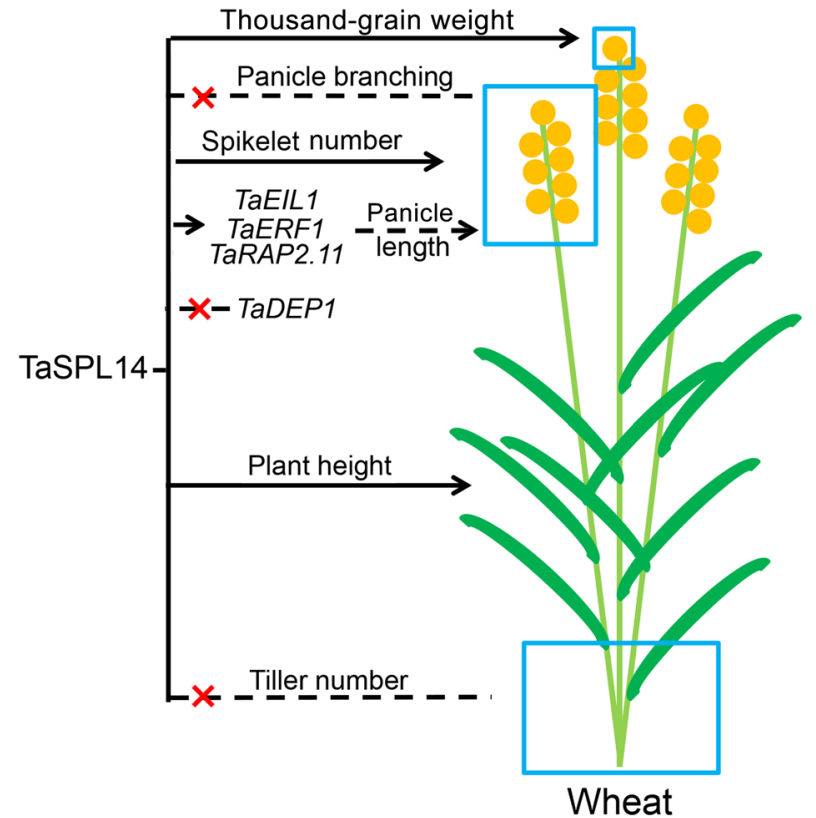

Fig. 5 A summary of the functional similarities and differences between TaSPL14 and OsSPL14. Similar to OsSPL14 in rice, TaSPL14 acts as a regulator in wheat that pleiotropically regulates plant height, panicle length, spikelet number, and thousand-grain weight. Unlike OsSPL14 in rice, TaSPL14 in wheat had no effect

Similar to OsSPL14, TaSPL14 acts as a major regulator of spike morphology. taspll4 plants showed a reduction in panicle length and spikelet number, but no alterations in panicle branching (Fig. 2c, d). OsSPL14 influences rice panicle architecture not only by regulating panicle length and spikelet number, but also by strongly affecting primary branching (Jiao et al. 2010; Miura et al. 2010; Kim et al. 2018). Therefore, TaSPL14 and OsSPL14 show functional similarly in regulating panicle length and spikelet number per panicle. Notably, OsSPL14 is a positive regulator of $D E P 1$, which is an important component of panicle architecture regulation (Lu et al. 2013). However, we observed that the expression level of TaDEPl was not affected in taspll4 plants compared with that in wild type (Suppl. Fig. S2), suggesting that TaSPL14 might regulate spike development via pathways involving genes other than $D E P 1$. We demonstrated that the expression levels of seven genes associated with ethylene response were significantly decreased in taspl14-\#13-4 as compared to the wild type, and that TaSPL14 could directly and dramatically activate the promoter activities of TaEILI, TaERF1, and TaRAP2.11. These data indicate that TaSPL14 might regulate spike development by regulating the ethyleneresponse pathway (Fig. 5).

In contrast to OSSPL14 in rice, TaSPL14 did not affect tillering in wheat. In rice, osspll4 mutants showed two contrasting tiller-number phenotypes: osspll4 mutants, carrying an amino-acid deletion in the OsSPL14 protein-coding sequence that maintained the activity of the protein, showed fewer tillers

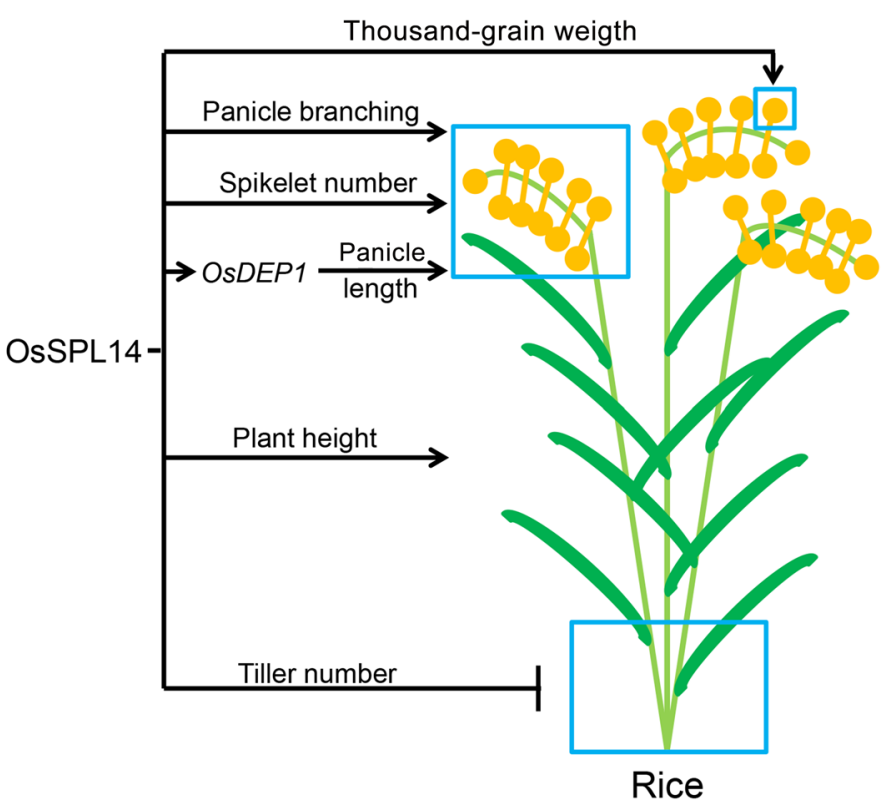

on panicle branching and tiller number. OsSPL14 regulates panicle length by positively activating $O S D E P 1$, but TaSPL14 might regulate wheat spike development via the ethylene-response-related genes TaEIL1, TaERF1, and TaRAP2.11 and not via TaDEP1

than wild type (Li et al. 2016); however, osspll4 mutants, with a frameshift mutation that might completely inactivate the OsSPL14 protein, had a dwarf phenotype and more tillers than wild type ( $\mathrm{Li}$ et al. 2016). Here, we observed that the tiller number of all TaSPL14 knock-out lines was similar to that in wild type at the heading stage in the field (Fig. 2f), indicating a functional difference between OSSPL14 and TaSPL14.

Three yield-positive $O S S P L 14$ alleles are known in rice ipal-1D, the WEALTHY FARMER'S PANICLE (WFP) epigenetic allele and the ipal-2D epigenetic allele all of which have been used in rice breeding (Jiao et al. 2010; Miura et al. 2010; Zhang et al. 2017). Introduction of the OSSPL14 ${ }^{\text {ipal }}$ allele into Xiushui11, a japonica rice variety, increased grain yield by $10 \%$ in the field (Jiao et al. 2010). Moreover, introgression of the OSSPL14 ${ }^{\mathrm{WFP}}$ allele into different rice varieties significantly increased the number of primary branches and grains per plant (Ashikari et al. 2005; Ashikari and Matsuoka 2006; Miura et al. 2010; Kim et al. 2018). JYZK-3 and JYZK-4 are two hybrid varieties that carry ipal-2D and show markedly improved yield performance in comparison with the control variety at different locations (Zhang et al. 2017). Thus, it is meaningful to identify natural variation related to the optimal expression levels of TaSPL14 in young panicles, which could be used to design and generate high-yielding new varieties using marker-assisted selection.

Author contribution statement Z.N., Q.S., Y.Y., and D.J. designed the project and all experiments; J.C., K.L., W.S., 
and N.Z. carried out the experiments and analyzed experimental data; M.X., H.P., and Z.H. were responsible for wheat transformation; J.C., Y.Y., and D.J contributed to the writing and revision of the manuscript. All authors read and approved the content of this manuscript.

\begin{abstract}
Acknowledgements We thank Dr. Qijun Chen (China Agricultural University) for providing the CRISPR-Cas9 vector (pBUE11) and Dr. Rentao Song (China Agricultural University) for help with transcriptional activity assays in N. benthamiana and for providing the pGreenII 0800-LUC expression vector.
\end{abstract}

Funding The National Natural Science Foundation of China (91935302) supported this work.

Data availability The datasets generated and analyzed during the current study are available in the NCBI (PRJNA629470) repository, https ://www.ncbi.nlm.nih.gov/Traces/study/?acc=PRJNA629470.

\section{Compliance with ethical standards}

Conflict of interest The authors declare that they have no conflict of interest.

Open Access This article is licensed under a Creative Commons Attribution 4.0 International License, which permits use, sharing, adaptation, distribution and reproduction in any medium or format, as long as you give appropriate credit to the original author(s) and the source, provide a link to the Creative Commons licence, and indicate if changes were made. The images or other third party material in this article are included in the article's Creative Commons licence, unless indicated otherwise in a credit line to the material. If material is not included in the article's Creative Commons licence and your intended use is not permitted by statutory regulation or exceeds the permitted use, you will need to obtain permission directly from the copyright holder. To view a copy of this licence, visit http://creativecommons.org/licenses/by/4.0/.

\section{References}

Appels R, Eversole K, Feuillet C, Keller B, Rogers J et al (2018) Shifting the limits in wheat research and breeding using a fully annotated reference genome. Science 361(6403):e7191

Ashikari M, Matsuoka M (2006) Identification, isolation and pyramiding of quantitative trait loci for rice breeding. Trends Plant Sci 11(7):344-350

Ashikari M, Sakakibara H, Lin S, Yamamoto T, Takashi T et al (2005) Cytokinin oxidase regulates rice grain production. Science 309(5735):741

Cardon G, Höhmann S, Klein J, Nettesheim K, Saedler H et al (1999) Molecular characterisation of the Arabidopsis SBP-box genes. Gene 237(1):91-104

Clavijo BJ, Venturini L, Schudoma C, Accinelli GG, Kaithakottil G et al (2017) An improved assembly and annotation of the allohexaploid wheat genome identifies complete families of agronomic genes and provides genomic evidence for chromosomal translocations. Genome Res 27(5):885-896

Dong H, Yan S, Liu J, Liu P, Sun J (2019) TaCOLD1 defines a new regulator of plant height in bread wheat. Plant Biotechnol J 17(3):687-699
Feng N, Song G, Guan J, Chen K, Jia M et al (2017) Transcriptome profiling of wheat inflorescence development from spikelet initiation to floral patterning identified stage-specific regulatory genes. Plant Physiol 174(3):1779-1794. https://doi.org/10.1104/pp.17.00310

Finn RD, Clements J, Eddy SR (2011) HMMER web server: interactive sequence similarity searching. Nucleic Acids Res 39(2):29-37

Guan Q, Yue X, Zeng H, Zhu J (2014) The protein phosphatase RCF2 and its interacting partner NAC019 are critical for heat stressresponsive gene regulation and thermotolerance in Arabidopsis. Plant Cell 26(1):438-453

Guo W, Yang H, Liu Y, Gao Y, Ni Z et al (2015) The wheat transcription factor TaGAMyb recruits histone acetyltransferase and activates the expression of a high-molecular-weight glutenin subunit gene. Plant J 84(2):347-359

Guo G, Liu X, Sun F, Cao J, Huo N et al (2018) Wheat miR9678 affects seed germination by generating phased siRNAs and modulating abscisic acid/gibberellin signaling. Plant Cell 30(4):796-814

Hellens RP, Allan AC, Friel EN, Bolitho K, Grafton K et al (2005) Transient expression vectors for functional genomics, quantification of promoter activity and RNA silencing in plants. Plant Methods 1(13): $1-14$

Huang X, Qian Q, Liu Z, Sun H, He S et al (2009) Natural variation at the $D E P 1$ locus enhances grain yield in rice. Nat Genet 41(4):494-497

Ishida Y, Tsunashima M, Hiei Y, Komari T (2015) Wheat (Triticum aestivum $\mathrm{L}$.) transformation using immature embryos. Methods Mol Biol 1223:189-198

Jiao Y, Wang Y, Xue D, Wang J, Yan M et al (2010) Regulation of OsSPL14 by OsmiR156 defines ideal plant architecture in rice. Nat Genet 42(6):541-544

Joshi NA, Fass JN (2011) Sickle: a sliding-window, adaptive, qualitybased trimming tool for FastQ files (Version 1.33) [Software]. Available at https://github.com/najoshi/sickle

Kerr SC, Beveridge CA (2017) IPA1: a direct target of SL signaling. Cell Res 27(10):1191-1192

Kim D, Pertea G, Trapnell C, Pimentel H, Kelley R et al (2013) TopHat2: accurate alignment of transcriptomes in the presence of insertions, deletions and gene fusions. Genome Biol 14(4):R36

Kim SR, Ramos JM, Hizon RJM, Ashikari M, Virk PS et al (2018) Introgression of a functional epigenetic OsSPL14 (WFP) allele into elite indica rice genomes greatly improved panicle traits and grain yield. Sci Rep 8(1):3833

Kuraparthy V, Sood S, Dhaliwal HS, Chhuneja P, Gill BS (2007) Identification and mapping of a tiller inhibition gene (tin3) in wheat. Theor Appl Genet 114(2):285-294

Li C, Lu S (2014) Molecular characterization of the SPL gene family in Populus trichocarpa. BMC Plant Biol 14:131. https://doi. org/10.1186/1471-2229-14-131

Li LF, Liu B, Olsen KM, Wendel JF (2015) A re-evaluation of the homoploid hybrid origin of Aegilops tauschii, the donor of the wheat D-subgenome. New Phytol 208(1):4-8

Li M, Li X, Zhou Z, Wu P, Fang M et al (2016) Reassessment of the four yield-related genes Gnla, DEP1, GS3, and IPAl in rice using a CRISPR/Cas9 System. Front Plant Sci 7:377

Ling HQ, Ma B, Shi X, Liu H, Dong L et al (2018) Genome sequence of the progenitor of wheat A subgenome Triticum urartu. Nature 557(7705):424-428

Liu M, Shi Z, Zhang X, Wang M, Zhang L et al (2019) Inducible overexpression of Ideal Plant Architecture1 improves both yield and disease resistance in rice. Nat Plants 5(4):389-400

Lu Z, Yu H, Xiong G, Wang J, Jiao Y et al (2013) Genome-wide binding analysis of the transcription activator ideal plant architecture 1 reveals a complex network regulating rice plant architecture. Plant Cell 25(10):3743-3759 
Minakuchi K, Kameoka H, Yasuno N, Umehara M, Luo L et al (2010) FINE CULM1 (FC1) works downstream of strigolactones to inhibit the outgrowth of axillary buds in rice. Plant Cell Physiol 51(7):1127-1135

Miura K, Ikeda M, Matsubara A, Song XJ, Ito M et al (2010) OsSPL14 promotes panicle branching and higher grain productivity in rice. Nat Genet 42(6):545-549

Ordonio RL, Matsuoka M (2017) New path towards a better rice architecture. Cell Res 27(10):1189-1190

Peng J, Richards DE, Hartley NM, Murphy GP et al (1999) 'Green revolution' genes encode mutant gibberellin response modulators. Nature 400(6741):256-651

Petersen G, Seberg O, Yde M, Berthelsen K (2006) Phylogenetic relationships of Triticum and Aegilops and evidence for the origin of the A, B, and D genomes of common wheat (Triticum aestivum). Mol Phylogenet Evol 39(1):70-82

Quinlan AR, Hall IM (2010) BEDTools: a flexible suite of utilities for comparing genomic features. Bioinformatics 26(6):841-842

Robinson MD, McCarthy DJ, Smyth GK (2010) edgeR: a Bioconductor package for differential expression analysis of digital gene expression data. Bioinformatics 26(1):139-140

Song X, Lu Z, Yu H, Shao G, Xiong J et al (2017) IPA1 functions as a downstream transcription factor repressed by D53 in strigolactone signaling in rice. Cell Res 27(9):1128-1141

Spielmeyer W, Richards RA (2004) Comparative mapping of wheat chromosome 1AS which contains the tiller inhibition gene (tin) with rice chromosome 5S. Theor Appl Genet 109(6):1303-1310

Takeda T, Suwa Y, Suzuki M, Kitano H, Tanaka MU et al (2003) The OsTB1 gene negatively regulates lateral branching in rice. Plant J 33(3):513-520

Vavilova V, Konopatskaia I, Kuznetsova AE, Blinov A, Goncharov NP (2017) DEP1 gene in wheat species with normal, compactoid and compact spikes. BMC Genet 18(1):106. https://doi. org/10.1186/s12863-017-0583-6

Wang B, Wang H (2017) IPA1: a new "Green Revolution" gene? Mol Plant 10(6):779-781

Wang J, Zhou L (2018) A single transcription factor promotes both yield and immunity in rice. Science 361(6406):1026-1028

Wang J, Yu H, Xiong G, Lu Z, Jiao Y (2017) Tissue-specific ubiquitination by IPA1 INTERACTING PROTEIN1 modulates IPA1 protein levels to regulate plant architecture in rice. Plant Cell 29(4):697-707
Xie K, Wu C, Xiong L (2006) Genomic organization, differential expression, and interaction of SQUAMOSA promoter-bindinglike transcription factors and microRNA156 in rice. Plant Physiol 142(1):280-293

Xing HL, Dong L, Wang ZP, Zhang HY, Han CY et al (2014) A CRISPR/Cas9 toolkit for multiplex genome editing in plants. BMC Plant Biol 14:327. https://doi.org/10.1186/s1287 0-014-0327-y

$\mathrm{Xu} \mathrm{H}$, Zhao M, Zhang Q, Xu Z, Xu Q (2016) The DENSE AND ERECT PANICLE 1 (DEP1) gene offering the potential in the breeding of high-yielding rice. Breeding Sci 66(5):659-667

Yan H, You Q, Tian T, Yi X, Liu Y et al (2017) agriGO v2.0: a GO analysis toolkit for the agricultural community. Nucleic Acids Res 45(1):122-129

Yang Z, Wang X, Gu S, Hu Z, Xu H et al (2008) Comparative study of SBP-box gene family in Arabidopsis and rice. Gene 407:1-11

Zhang J, Wu J, Liu W, Lu X, Yang X et al (2013) Genetic mapping of a fertile tiller inhibition gene, ftin, in wheat. Mol Breeding 31(2):441-449

Zhang B, Liu X, Zhao G, Mao X, Li A, Jing R (2014) Molecular characterization and expression analysis of Triticum aestivum squamosa-promoter binding protein-box genes involved in ear development. J Integr Plant Biol 56(6):571-581

Zhang L, Yu H, Ma B, Liu G, Wang J et al (2017) A natural tandem array alleviates epigenetic repression of IPAl and leads to superior yielding rice. Nat Commun 8:14789

Zhang W, Zhang M, Zhu X, Cao Y, Sun Q et al (2018) Molecular cytogenetic and genomic analyses reveal new insights into the origin of the wheat B genome. Theor Appl Genet 131(2):365-375

Zhao M, Sun J, Xiao Z, Cheng F, Xu H et al (2016) Variations in DENSE AND ERECT PANICLE 1 (DEPI) contribute to the diversity of the panicle trait in high-yielding japonica rice varieties in northern China. Breeding Sci 66(4):599-605

Zheng M, Liu X, Lin J, Liu X, Wang Z et al (2019) Histone acetyltransferase GCN5 contributes to cell wall integrity and salt stress tolerance by altering the expression of cellulose synthesis genes. Plant J 97(3):587-602

Publisher's Note Springer Nature remains neutral with regard to jurisdictional claims in published maps and institutional affiliations.

\section{Authors and Affiliations}

\section{Jie Cao ${ }^{1} \cdot$ Kaiye Liu ${ }^{1} \cdot$ Wanjun Song ${ }^{1} \cdot$ Jianing Zhang ${ }^{1} \cdot$ Yingyin Yao ${ }^{1} \cdot$ Mingming Xin ${ }^{1} \cdot$ Zhaorong Hu$^{1} \cdot$ Huiru Peng ${ }^{1}$. Zhongfu Ni ${ }^{1}$. Qixin Sun ${ }^{1}$ · Jinkun $\mathrm{Du}^{1}$ (])}

Jie Cao
cao2014jie@163.com

Kaiye Liu

kaiyeliu@cau.edu.cn

Wanjun Song

songwanjun112@163.com

Jianing Zhang

zjianing6@163.com

Yingyin Yao

yingyin@cau.edu.cn

Mingming Xin

mingmingxin@cau.edu.cn

Zhaorong $\mathrm{Hu}$

zrhu@cau.edu.cn
Huiru Peng

penghuiru@cau.edu.cn

Zhongfu Ni

nizf@cau.edu.cn

Qixin Sun

qxsun@cau.edu.cn

1 State Key Laboratory for Agrobiotechnology and Key Laboratory of Crop Heterosis and Utilization (MOE) and Beijing Key Laboratory of Crop Genetic Improvement, China Agricultural University, Beijing 100193,

People's Republic of China 